\title{
EXACT STRONG LAWS FOR FUNCTIONS FROM THE BIVARIATE PARETO DISTRIBUTION
}

\begin{abstract}
ANDRÉ ADLER
Department of Applied Mathematics, Illinois Institute of Technology, Chicago, Illinois, 60616, USA. E-mail: adler@iit.edu

\|\|

Abstract

We examine several functions from the bivariate Pareto distribution and obtain surprising results in regards to almost sure convergence. We look at the usual functions, such as the sum, the difference, the ratio, the maximum and the minimum as well as the ratio of the max and the min. What happens here in the nonindependent situation is quite different from what we have seen before.
\end{abstract}

\section{Introduction}

In this paper we observe the bivariate Pareto. The accepted definition can be traced back to [6]

$$
f(x, y)=\frac{2}{(x+y-1)^{3}}
$$

where both $x \geq 1$ and $y \geq 1$. For more on this distribution one should read 4]. Arnold is an expert on the Pareto. We will examine the usual suspects and the results are not what one would expect. The ratio of exponentials and Paretos and uniforms and many other sets of independent random variables did produce an Exact Strong Law. There are many papers on that topic, just a few are [2], [5], 7], [8] and [9]. In this nonindependent setting that is not the case. And it turns out that many of the other functions we observe do have an unusual strong law, even the minimum, which never happened

Received March 31, 2021.

AMS Subject Classification: 60F05, 60F15.

Key words and phrases: Almost sure convergence, strong laws of large numbers, exact strong laws, central limit theorems, bivariate Pareto distribution. 
in any other setting. The odd thing here is that the ratio doesn't produce a strange strong law. It produces a strange Central Limit Theorem.

We need to say that the constant $C$, used in the proofs denotes a generic real number that is not necessarily the same in each appearance. It is used as an upper bound in order to establish the convergence of our various series. Also, we define $\lg x=\ln (\max \{e, x\})$ to avoid dividing by zero.

\section{The Sum}

This result is not surprising since the marginal density of $X$ is $f_{X}(x)=$ $x^{-2} I(x \geq 1)$ and the marginal density of $Y$ is $f_{Y}(y)=y^{-2} I(y \geq 1)$. Each of these has an Exact Strong Law. So, we will start with this easy example and move on to more interesting ones. The joint distribution of the sum is

$$
F_{X+Y}(a)=P\{X+Y \leq a\}=\int_{1}^{a-1} \int_{1}^{a-x} \frac{2 d y d x}{(x+y-1)^{3}}=1-\frac{2 a-3}{(a-1)^{2}} .
$$

We could have used the transformation of $S=X+Y$ and $W=X$, but in other situations that technique is too messy. However, if one wants to obtain the density via Jacobians the joint density of $S$ and $W$ is

$$
f(s, w)=\frac{2}{(s-1)^{3}}
$$

where $1 \leq w \leq s-1$. Thus

$$
f(s)=\int_{1}^{s-1} \frac{2}{(s-1)^{3}}=\frac{2(s-2)}{(s-1)^{3}}
$$

which agrees with (11).

Theorem 1. Let $\left\{\left(X_{i}, Y_{i}\right), i \geq 1\right\}$ be i.i.d. bivariate Pareto random variables. Then for $\alpha>-2$ we have

$$
\lim _{n \rightarrow \infty} \frac{\sum_{i=1}^{n} \frac{(\lg i)^{\alpha}}{i}\left(X_{i}+Y_{i}\right)}{(\lg n)^{\alpha+2}}=\frac{2}{\alpha+2} \quad \text { almost surely. }
$$

Proof. This follows from Example 2 of [1], since

$$
1-F_{X+Y}(a)=P\{X+Y>a\} \sim 2 / a
$$

completing this proof. 


\section{The Difference}

Due to symmetry we cannot get an Exact Strong Law for the difference, but we can obtain a strong law of large numbers. And we can use that to obtain an Exact Strong Law for the absolute difference.

Theorem 2. Let $\left\{\left(X_{i}, Y_{i}\right), i \geq 1\right\}$ be i.i.d. bivariate Pareto random variables. Then for $\alpha>-2$ we have

$$
\lim _{n \rightarrow \infty} \frac{\sum_{i=1}^{n} \frac{(\lg i)^{\alpha}}{i}\left(X_{i}-Y_{i}\right)}{(\lg n)^{\alpha+2}}=0 \quad \text { almost } \text { surely. }
$$

Proof.. We need to find the distribution function of the difference. Here the random variable, $X-Y$ can be negative, so there are two cases. If $a<0$, then

$$
F_{X-Y}(a)=P\{X-Y \leq a\}=\int_{1-a}^{\infty} \int_{1}^{y+a} \frac{2 d x d y}{(x+y-1)^{3}}=\frac{1}{2(1-a)}
$$

And if $a \geq 0$, then

$$
F_{X-Y}(a)=P\{X-Y \leq a\}=\int_{1+a}^{\infty} \int_{1}^{x-a} \frac{2 d y d x}{(x+y-1)^{3}}=\frac{1}{2(1+a)}
$$

Hence

$$
F_{X-Y}(a)= \begin{cases}\frac{1}{2(1-a)}, & \text { if } a<0 \\ \frac{1}{2(1+a)}, & \text { if } a \geq 0\end{cases}
$$

So, the limit is zero by Theorem 2 of [1].

We did obtain a strong law of large numbers, but the limit wasn't interesting since the two tails were equal. However, if we look at $|X-Y|$, then we have an Exact Strong Law.

Theorem 3. Let $\left\{\left(X_{i}, Y_{i}\right), i \geq 1\right\}$ be i.i.d. bivariate Pareto random variables. Then for $\alpha>-2$ we have

$$
\lim _{n \rightarrow \infty} \frac{\sum_{i=1}^{n} \frac{(\lg i)^{\alpha}}{i}\left|X_{i}-Y_{i}\right|}{(\lg n)^{\alpha+2}}=\frac{1}{\alpha+2} \quad \text { almost surely. }
$$


Proof. In order to obtain the distribution function for $|X-Y|$ we use

$$
\begin{aligned}
1-F_{|X-Y|}(a) & =P\{|X-Y|>a\}=P\{X-Y>a\}+P\{X-Y<-a\} \\
& =1-F_{X-Y}(a)+F_{X-Y}(-a)=\frac{1}{1+a} \sim \frac{1}{a} .
\end{aligned}
$$

So, using Example 2 from [1] the conclusion follows.

\section{Order Statistics}

The results here are somewhat surprising. One would expect an Exact Strong Law for the maximum, but not the minimum.

Theorem 4. Let $\left\{\left(X_{i}, Y_{i}\right), i \geq 1\right\}$ be i.i.d. bivariate Pareto random variables. Then for $\alpha>-2$ we have

$$
\lim _{n \rightarrow \infty} \frac{\sum_{i=1}^{n} \frac{(\lg i)^{\alpha}}{i} \max \left\{X_{i}, Y_{i}\right\}}{(\lg n)^{\alpha+2}}=\frac{3}{2(\alpha+2)} \quad \text { almost surely. }
$$

Proof. For all $a \geq 1$

$$
F_{\max \{X, Y\}}(a)=P\{X \leq a, Y \leq a\}=\int_{1}^{a} \int_{1}^{a} \frac{2 d y d x}{(x+y-1)^{3}}=1-\frac{3 a-2}{a(2 a-1)} .
$$

Hence

$$
1-F_{\max \{X, Y\}}(a) \sim \frac{3}{2 a} .
$$

Once again using Example 2 from [1] the conclusion follows.

Theorem 5. Let $\left\{\left(X_{i}, Y_{i}\right), i \geq 1\right\}$ be i.i.d. bivariate Pareto random variables. Then for $\alpha>-2$ we have

$$
\lim _{n \rightarrow \infty} \frac{\sum_{i=1}^{n} \frac{(\lg i)^{\alpha}}{i} \min \left\{X_{i}, Y_{i}\right\}}{(\lg n)^{\alpha+2}}=\frac{1}{2(\alpha+2)} \quad \text { almost } \text { surely. }
$$

Proof. For all $a \geq 1$

$$
1-F_{\min \{X, Y\}}(a)=P\{X>a, Y>a\}=\int_{a}^{\infty} \int_{a}^{\infty} \frac{2 d y d x}{(x+y-1)^{3}}=\frac{1}{2 a-1} .
$$


Hence

$$
1-F_{\min \{X, Y\}}(a) \sim \frac{1}{2 a} .
$$

Once again using Example 2 from [1] the conclusion follows.

There is also an unusual limit theorem for the ratio of these two statistics. What is surprising is that this random variable does have a first, but not a second moment. This and the usual ratio will be covered in the last section.

\section{Ratios and the Central Limit Theorem}

This is the most surprising of all. When we examined ratios of independent Paretos or uniforms or even exponentials random variables, we always had an Exact Strong Law. This was extended in [5]. That does not happen in the bivariate Pareto setting. Here the classic strong law holds, but in this situation we do obtain an unusual Central Limit Theorem, quite reminiscent of [2]. We will use Theorem 4 from [3].

There are three conditions that we need to meet in order to apply that theorem. The first one is that

$$
G(a)=\int_{0}^{a} 2 t P\{|W|>t\} d t
$$

is slowly varying. The other two are

$$
G\left(\frac{B_{n}}{\min _{1 \leq i \leq n} a_{i}}\right) \sim G\left(\frac{B_{n}}{\max _{1 \leq i \leq n} a_{i}}\right)
$$

and for all $\epsilon>0$

$$
\sum_{i=1}^{n} P\left\{|W|>\epsilon B_{n} / a_{i}\right\}=o(1)
$$

where $a_{i}$ are our weights, $B_{n}$ is our norming sequence and $W$ is our random variable of interest. The norming sequence is derived from $B_{n}^{2} \sim$ $n G\left(B_{n}\right)$. We examine the limiting behaviour of both $W=Y / X$ and $W=$ $\max \{X, Y\} / \min \{X, Y\}$. 
Theorem 6. Let $\left\{\left(X_{i}, Y_{i}\right), i \geq 1\right\}$ be i.i.d. bivariate Pareto random variables. If $\alpha<1 / 2$, then

$$
\frac{\sum_{i=1}^{n}(\lg i)^{\alpha}\left[\frac{Y_{i}}{X_{i}}-\frac{3}{2}\right]}{\sqrt{n \lg n}} \stackrel{d}{\rightarrow} N(0,1)
$$

Proof. We need to find the distribution of $Y / X$. If $0 \leq a<1$, then

$$
F_{Y / X}(a)=P\{Y \leq a X\}=\int_{1 / a}^{\infty} \int_{1}^{a x} \frac{2 d y d x}{(x+y-1)^{3}}=\frac{a^{2}}{a+1}
$$

and if $a \geq 1$, then

$$
1-F_{Y / X}(a)=P\{Y>a X\}=\int_{a}^{\infty} \int_{1}^{y / a} \frac{2 d x d y}{(x+y-1)^{3}}=\frac{1}{a(a+1)}
$$

so, the density of this random variable is

$$
f_{Y / X}(a)= \begin{cases}0, & \text { if } a<0 \\ \frac{a(a+2)}{(a+1)^{2}}, & \text { if } 0 \leq a<1 \\ \frac{2 a+1}{a^{2}(a+1)^{2}}, & \text { if } a \geq 1\end{cases}
$$

and its expectation is $3 / 2$. Using (21) we have

$$
G(a)=\int_{0}^{a} 2 t P\{Y / X>t\} d t \sim \int_{1}^{a} \frac{2 d t}{t+1} \sim 2 \lg a .
$$

One solution of $B_{n}^{2} \sim n G\left(B_{n}\right)$ is $B_{n}=\sqrt{n \lg n}$. Equation (3) holds with $a_{i}=(\lg i)^{\alpha}$. Finally, we establish (4). Let $\epsilon>0$

$$
\begin{aligned}
\sum_{i=1}^{n} P\left\{Y / X>\epsilon B_{n} / a_{i}\right\} & <C \sum_{i=1}^{n} \int_{\epsilon B_{n} / a_{i}}^{\infty} \frac{d t}{t^{3}} \\
& <\frac{C \sum_{i=1}^{n} a_{i}^{2}}{B_{n}^{2}}=\frac{C \sum_{i=1}^{n}(\lg i)^{2 \alpha}}{n \lg n} \rightarrow 0
\end{aligned}
$$

since $\alpha<1 / 2$, concluding this proof.

We finish with a central limit theorem for the ratio of our two order statistics. One would expect an Exact Strong Law here since both the minimum and maximum produced one, but that does not happens with this distribution. 
Theorem 7. Let $\left\{\left(X_{i}, Y_{i}\right), i \geq 1\right\}$ be i.i.d. bivariate Pareto random variables. If $\alpha<1 / 2$, then

$$
\frac{\sum_{i=1}^{n}(\lg i)^{\alpha}\left[\frac{\max \left\{X_{i}, Y_{i}\right\}}{\min \left\{X_{i}, Y_{i}\right\}}-(1+2 \lg 2)\right]}{\sqrt{2 n \lg n}} \stackrel{d}{\rightarrow} N(0,1) .
$$

Proof. Set $W=\max \{X, Y\} / \min \{X, Y\}$ and let $a \geq 1$, then

$$
\begin{aligned}
1-F_{W}(a) & =P\{Y>a X\}+P\{Y \leq X / a\} \\
& =\int_{a}^{\infty} \int_{1}^{y / a} \frac{2 d x d y}{(x+y-1)^{3}}+\int_{a}^{\infty} \int_{1}^{x / a} \frac{2 d y d x}{(x+y-1)^{3}} \\
& =2 \int_{a}^{\infty} \int_{1}^{y / a} \frac{2 d x d y}{(x+y-1)^{3}}=\frac{2}{a(a+1)} .
\end{aligned}
$$

The density is

$$
f_{W}(a)=\frac{2(2 a+1)}{a^{2}(a+1)^{2}}
$$

where $a \geq 1$ and

$$
G(a)=\int_{0}^{a} 2 t P\{W>t\} d t=\int_{1}^{a} \frac{4 d t}{t+1} \sim 4 \lg a .
$$

A solution of $B_{n}^{2} \sim n G\left(B_{n}\right)$ is $B_{n}=\sqrt{2 n \lg n}$. Once again (3) holds with $a_{i}=(\lg i)^{\alpha}$. Let $\epsilon>0$

$$
\begin{aligned}
\sum_{i=1}^{n} P\left\{W>\epsilon B_{n} / a_{i}\right\} & <C \sum_{i=1}^{n} \int_{\epsilon B_{n} / a_{i}}^{\infty} \frac{d t}{t^{3}} \\
& <\frac{C \sum_{i=1}^{n} a_{i}^{2}}{B_{n}^{2}}=\frac{C \sum_{i=1}^{n}(\lg i)^{2 \alpha}}{2 n \lg n} \rightarrow 0
\end{aligned}
$$

since $\alpha<1 / 2$. Finally, the expectation is

$$
E(W)=\int_{1}^{\infty} \frac{2(2 t+1) d t}{t(t+1)^{2}}=1+2 \lg 2
$$

which concludes this proof. 


\section{The Product}

The one function that doesn't produce a strange limit theorem is the product. Here $P\{X Y>a\}$ is equal to

$1-P\{X Y \leq a\}=1-\int_{1}^{a} \int_{1}^{a / x} \frac{2 d y d x}{(x+y-1)^{3}}=\frac{1}{a}+\int_{1}^{a}\left(x+\frac{a}{x}-1\right)^{2} d x \sim \frac{\pi}{4 \sqrt{a}}$.

This grows too fast to achieve an Exact Strong Law. So the product fails to give an unusual limit theorem, but all the other classic functions of $X$ and $Y$ do allow us to obtain new results.

\section{References}

1. A. Adler, Exact Strong Laws, Bull. Inst. Math. Acad. Sinica, 28 (2000), no. 3, 141-165.

2. A. Adlerand and P. Matula, Unusual limit theorems for the two tailed Pareto distribution, Probab. Math. Stat., (2020), to appear.

3. A. Adler and A. Rosalsky, Some generalized central limit theorems for weighted sums with infinite variance, Calcutta Statist. Assoc. Bull., 38 (1989), 27-42. doi.org/10.1177/0008068319890102

4. B. C. Arnold, Pareto Distributions, Monographs on Statistics and Applied Probability 140, second edition, CRC Press, Chapman and Hall, 2015.

5. P. Matula, P. Kurasinski, H. Naderi, A. Adler, A note on exact laws of large numbers for asymmetric Pareto-type distributions with applications to ratios of random variables, Bull. Inst. Math. Acad. Sinica, 15 (2020), no. 2, 177-186.

DOI: 10.21915/BIMAS.2020205

6. K. V. Mardia, Multivariate Pareto Distributions, Ann. Math. Statist., 33 (1962), No. 3, 1008-1015. https://www.jstor.org/stable/2237876

7. H. Xu, X. Li, W. Yang, F. Xu, Laws of large numbers with infinite mean, Journal Math. Inequal., 13 (2019), no. 2, 335-349.

8. S. Xu, C. Mei and Y. Miao, Limit theorems for ratios of order statistics from uniform distributions, Journal Inequalities and Applications, (2019),

DOI: $10.1186 / \mathrm{s} 13660-019-2256-7$.

9. W. Yang, L. Yang, D. Wei and S. Hu, The laws of large numbers for Pareto-type random variables with infinite means, Communications in Statistics - Theory and Methods, 48 (2019), no.12, 3044-3054. 